\title{
On the controllability of bilinear quantum systems
}

\author{
Gabriel TURINICI \\ ASCI-CNRS Laboratory, Bat. 506, Université Paris Sud, 91405 Orsay Cedex
}

\begin{abstract}
We present in this paper controllability results for quantum systems interacting with lasers. A negative result for infinite dimensional spaces serves as a starting point for a finite dimensional analysis. We show that under physically reasonable hypothesis in such systems we can control the population of the eigenstates. Applications are given for a five-level system.
\end{abstract}

\section{Introduction}

Controlling chemical reactions at the quantum level was a long-lasting goal for the Chemists (cf. [4], [8], [9], [11], [12], [13], [15]) from the very beginning of the laser technology. Indeed, due to the subtle nature of the interactions involved, this kind of manipulation is expected to allow on the one hand for much efficient and finer control than classical tools (temperature, pressure, catalyzers ...) and on the other hand for new reactions and/or products to be obtained.

The first experiments have shown that designing the laser pulse able to steer the system to the desired target state is a rather difficult task that physical intuition alone cannot accomplish. It is only recently that tools coming from the control theory began to give satisfactory results in some particular cases; finding the optimal electric field is now treated by numerical methods and new models are sought after that be also reliable and cheap from a computational point of view.

A legitimate question arises in this context: what quantum states can be attained using such an external field? Some answers are given below. 


\section{Infinite dimensional controllability}

Our purpose is to control the equations that govern the time evolution of quantum systems. Let consider such a system (isolated from the outer world for the moment) whose internal Hamiltonian is $H_{0}$ that is prepared in the initial state $\Psi_{0}(x)$; its dynamics obeys the Time Dependent Schrödinger Equation. Denoting by $\Psi(x, t)$ the state at the time $t$ one can write the evolution equations for the free system:

$$
\left\{\begin{array}{l}
i \hbar \frac{\partial}{\partial t} \Psi(x, t)=H_{0} \Psi(x, t) \\
\Psi(x, t=0)=\Psi_{0}(x),\left\|\Psi_{0}\right\|_{L^{2}\left(\mathbf{R}^{\gamma}\right)}=1
\end{array}\right.
$$

In the presence of external interactions that for us will be an electric field created by a laser and modeled by a laser intensity $\epsilon(t) \in \mathbf{R}$ and by a certain time independent dipole moment operator ${ }^{1} \mathcal{B}$ the (controlled) dynamical equations reads:

$$
\left\{\begin{array}{l}
i \hbar \frac{\partial}{\partial t} \Psi_{\epsilon}(x, t)=H_{0} \Psi_{\epsilon}(x, t)-\epsilon(t) \mathcal{B} \Psi_{\epsilon}(x, t)=H \Psi_{\epsilon}(x, t) \\
\Psi_{\epsilon}(x, t=0)=\Psi_{0}(x)
\end{array}\right.
$$

The goal is to find (if any) a final time $T$ and a finite energy laser pulse $\epsilon(t), \epsilon(t) \in L^{2}([0, T])$ able to steer the system from $\Psi_{0}(x)$ to some predefined target $\Psi_{\epsilon}(x, T)=\Psi_{\text {target }}(x)$.

Note that $\Psi_{\epsilon}(x, t)$ is evolving on the unit sphere $S(0,1)$ of $L^{2}\left(\mathbf{R}^{\gamma}\right)$ :

$$
S(0,1)=\left\{f \in L^{2}\left(\mathbf{R}^{\gamma}\right) ;\|f\|_{L^{2}\left(\mathbf{R}^{\gamma}\right)}=1\right\}
$$

Indeed one can easily prove that the $L^{2}$ norm of $\Psi_{\epsilon}$ is conserved throughout the evolution:

$$
\left\|\Psi_{\epsilon}(x, t)\right\|_{L_{x}^{2}\left(\mathbf{R}^{\gamma}\right)}=\left\|\Psi_{0}\right\|_{L^{2}\left(\mathbf{R}^{\gamma}\right)}, \forall t>0 .
$$

Let us point out some simple (but important) remarks before carrying on the analysis of these equations. Firstly in what the target state is concerned it follows by the incertitude principle that one will never be able to experimentally verify, neither exploit, the exact controllability. In fact even if one method gives exactly the desired target state $\Psi_{\text {target }}$ the free evolution (i.e. when laser is switched off $\epsilon(t)=0, t \geq T)$ of the quantum system instantaneously modifies this state (by a time dependent phase factor if $\Psi_{\text {target }}$ is an eigenfunction of $H_{0}$ and by the (1) formula in general).

\footnotetext{
${ }^{1}$ Of course, depending on the problem at hand, one may sometime choose to go beyond this first-order, bilinear term when describing the interaction between the laser and the system, cf. [5], [6].
} 
In this context a first negative controllability result is therefore not really restrictive. In fact using compacity arguments as those in [1] we can prove the following ${ }^{2}$ :

Theorem 1 Let $\mathcal{B}$ be a bounded operator from $H_{x}^{2}\left(\mathbf{R}^{\gamma}\right)$ to itself and let $H_{0}$ generate a $C^{0}$ semigroup of bounded linear operators on $H_{x}^{2}\left(\mathbf{R}^{\gamma}\right)$. Denote by $\Psi_{\epsilon}(x, t)$ the solution of (2). Then the set of attainable states from $\Psi_{0}$ defined by

$$
\mathcal{A S}=\cup_{T>0}\left\{\Psi_{\epsilon}(x, T) ; \epsilon(t) \in L^{2}([0, T])\right\}
$$

is contained in a countable union of compact subsets of $H_{x}^{2}\left(\mathbf{R}^{\gamma}\right)$. In particular its complement with respect to $S(0,1): \mathcal{N}=S(0,1) \backslash \mathcal{A S}$ is everywhere dense on $S(0,1)$. The same holds true for the complement with respect to $S(0,1) \cap H_{x}^{2}\left(\mathbf{R}^{\gamma}\right)$.

Proof. To prove the first part of the theorem one applies Thm. 3.6 from [1] on the space $H_{x}^{2}\left(\mathbf{R}^{\gamma}\right)$ for the operators $-i H_{0}$ and $-i \mathcal{B}$ (and restricts $\epsilon(t)$ to $L^{2}$ functions).

Note that for any compact subset $K$ of $X$

$$
[0, n] \cdot K=\{r f ; 0 \leq r \leq n, f \in K\}
$$

is also compact. Applying this to the compact components $K$ of $\mathcal{A S}$ one notes that

$$
\cup_{r \geq 0} r \mathcal{A S}=\cup_{n \in \mathbf{N}^{*}}[0, n] \cdot \mathcal{A S}
$$

is also a countable union of compacts subsets of $H_{x}^{2}\left(\mathbf{R}^{\gamma}\right)$. It follows by the Baire category theorem that $\cup_{r>0} r \mathcal{A S}$ has dense complement in $H_{x}^{2}\left(\mathbf{R}^{\gamma}\right)$; in particular the complement of $\mathcal{A} \mathcal{S}$ with respect to $S(0,1) \cap H_{x}^{2}\left(\mathbf{R}^{\gamma}\right)$ has to be everywhere dense on $S(0,1) \cap H_{x}^{2}\left(\mathbf{R}^{\gamma}\right)$.

Given this result one may either study the controllability with respect to a finite number of moments or the controllability of the corresponding finite dimensional system. Is the second analysis that we chose to pursue in this paper.

\footnotetext{
${ }^{2}$ We refer to [7] for a different view on this issue. Let us point out however that their analysis is done on piecewise constant functions which may not always carry physical meaning for our problem; in particular one may prove controllability in this class but realize (by the theorem we present here) that this controllability requires infinite $L^{2}$ norm and therefore infinite laser energy.
} 


\section{$3 \quad$ Finite dimensional controllability}

Let then $D=\left\{\Psi_{i}(x) ; i=1, \ldots, N\right\}$ be an orthonormal basis for a finite dimensional sub-space of $L^{2}\left(\mathbf{R}^{\gamma}\right)$ that we are interested $i^{3}$ and $A$ and $B$ be the matrices of the operators $H_{0}$ and $\mathcal{B}$ respectively, with respect to this base ${ }^{4}$.

Let us denote by $C=\left(c_{i}\right)_{i=1}^{N}$ the coefficients of $\Psi_{i}(x)$ in the formula of the evolving state $\Psi(t, x)=\sum_{i=1}^{N} c_{i}(t) \Psi_{i}(x)$; then the equations (2) reads

$$
\begin{gathered}
\left\{\begin{array}{l}
i \hbar \frac{\partial}{\partial t} C_{\epsilon}=A C_{\epsilon}-\epsilon(t) B C_{\epsilon} \\
C_{\epsilon}(t=0)=C_{0}
\end{array}\right. \\
C_{0}=\left(c_{0 i}\right)_{i=1}^{N}, c_{0 i}=\int_{\mathbf{R}^{\gamma}} \Psi_{0} \Psi_{i} d x
\end{gathered}
$$

The controllability of (5) has been dealt with in the literature (cf. [10]) by reducing the problem to the controllability of a system posed on the space of the unitary matrices of dimension $N$. This approach has the benefit of granting us access to the general tools and results on bilinear controllability on Lie groups. However it does not correspond to a physical necessity and therefore gives criterions not so easy to verify; moreover all the results one can obtain this way give only sufficient conditions for exact controllability (due to the reduction above which is restrictive). Finally there exists a class of simple quantum systems controllable (in a sense to be defined further on) that do not verify the criteria emerging from the Lie group analysis.

We have therefore judged instructive to study this issue taking into account the specificity of the quantum framework; we were thus lead into identifying necessary and sufficient conditions for the finite dimensional controllability.

In the case of our modeling ${ }^{3}{ }^{4}$ the $A$ matrix is diagonal and $B$ is symmetrical and has null diagonal elements ${ }^{5}$. Let us denote by $\lambda_{i}, i=1, . ., N$ the diagonal elements of $A$ (the energies of the states $\Psi_{i}$ ).

Before presenting our controllability results we have to introduce the first elements required to explain our controllability concept. As it was previously seen the system evolves on the unit sphere of $L_{x}^{2}\left(\mathbf{R}^{\gamma}\right)$ which in finite dimensional representation reads:

$$
\sum_{i=1}^{N}\left|c_{\epsilon i}(t)\right|^{2}=1, \forall t \geq 0
$$

\footnotetext{
${ }^{3}$ This space is given by our model and the functions $\Psi_{i}(x)$ are usually the first eigenfunctions of $H_{0}$ constructed by a prior computation or by a modeling based on observations.

${ }^{4}$ We suppose in the begining that $\mathcal{B}$ is such that $B_{i i}=0, i=1, . ., N$; for the general case see the appendix.

${ }^{5}$ see the appendix for the general case
} 
From (5) one notices that when the system is evolving freely $((5)$ with $\epsilon(t)=0)$ the (relative) phases of the coefficients $c_{\epsilon i}(t)$ change but not the populations of the eigenstates. We will therefore study only the population transfer between eigenstates i.e. only changes in $\left|c_{i}(t)\right|^{2}$. We call population distribution for the system (5) any $N$-tuple $d \in \mathbf{R}^{N}$ such that

$$
\sum_{i=1}^{N} d_{i}^{2}=1, d_{i} \geq 0, i=1, \ldots, N
$$

We will also say that we can reach the population distribution d from the initial state $C_{0}$ if for any $\eta>0$ there exists a final time $T_{d}>0$ and an electric field $\epsilon(t) \in L^{2}\left(\left[0, T_{d}\right]\right)$ such that the solution of (5) satisfy $\left.|| c_{\epsilon k}\left(T_{d}\right)\right|^{2}-d_{k}^{2} \mid<\eta$. If this is also true for $\eta=0$ then we say that we can exactly reach the population distribution $d$ from the initial state $C_{0}$.

\section{Transfer graph and necessary conditions}

According to the physical intuition that we will support in the following by mathematical arguments, the $B$ matrix describes the population flow among different eigenstates of the system. In order to formalize this idea we associate to the system some graph $G=(V, E)$ called the transfer graph. We define the set $V$ of vertices as the set of eigenstates $\Psi_{i}$ and the set of edges $E$ as the set of all pairs of eigenstates coupled by the matrix $B$. Since $B$ is symmetrical we can consider $G$ non-oriented:

$$
G=(V, E): \quad V=\left\{\Psi_{1}, \ldots, \Psi_{n}\right\} \quad E=\left\{\left(\Psi_{i}, \Psi_{j}\right) ; B_{i j} \neq 0\right\}
$$

Let us decompose this graph into connected components $G_{\alpha}=\left(V_{\alpha}, E_{\alpha}\right)$, $a=1, . ., K$. Note that this decomposition corresponds to a bloc-diagonal structure of the matrix B (modulo some permutations on the indices). Using this decomposition one can write new conservation laws for each connected component:

$$
\sum_{\left\{i ; \Psi_{i} \in V_{\alpha}\right\}}\left|c_{\epsilon i}(t)\right|^{2}=\text { constant }, \forall t>0, \alpha=1, . ., K
$$

In order to justify (10) one checks by the definition of $G$ and using equations (5) that for all $\alpha=1, . ., K$ :

$$
i \hbar \frac{\partial}{\partial t} \sum_{\left\{i ; \Psi_{i} \in V_{\alpha}\right\}}\left|c_{\epsilon i}(t)\right|^{2}=0
$$

This allows us to give necessary conditions for controllability: 
Lemma 1 If one can reach the population distribution d from the initial configuration $C_{0}$ then

$$
\sum_{\left\{i ; \Psi_{i} \in V_{\alpha}\right\}}\left|c_{0 i}\right|^{2}=\sum_{\left\{i ; \Psi_{i} \in V_{\alpha}\right\}} d_{i}^{2}, \quad \alpha=1, . ., K
$$

Definition 1 We say that the population distribution of the system (5) is controllable if for any initial state $C_{0}$ and any population distribution $d$ that satisfy (12) it is possible to reach $d$ from the initial configuration $C_{0}$.

\section{Controllability results}

Denote $\omega_{k l}=\lambda_{k}-\lambda_{l}, k, l=1, \ldots, N$. To ease the notations we will be working in atomic units $(\hbar=1)$. Let us introduce the following hypothesis:

$\mathbf{H}$ The components $G_{\alpha}, \alpha=1, . ., K$ of $G$ remain connected after elimination of all edge pairs $\left(\Psi_{i}, \Psi_{j}\right),\left(\Psi_{a}, \Psi_{b}\right)$ such that $\omega_{i j}=\omega_{a b}$ (degenerate transitions).

\subsection{Local exact controllability}

Theorem 2 Let $d_{0}$ be the population distribution associated to the initial state $C_{0}: d_{0}=\left(\left|c_{0 i}\right|\right)_{i=1, \ldots, N}$. Suppose $d_{0 i} \neq 0, i=1, \ldots, N$ and that the hypothesis $(H)$ is verified. Then there exists an open neighborhood $D$ of $d_{0}$ on the surface of $\mathbf{R}^{N}$ given by the necessary conditions (12) endowed with the canonical topology such that one can exactly reach any population distribution d in $D$ from $C_{0}$.

Remark 1 The conditions $d_{0 i} \neq 0, i=1, \ldots, N$ are just technicalities needed in the proof. Note that if some $d_{0 i}=0$ one have to take care when choosing the good target set to expect exact controllability into, since there is no reason to hope in (exactly) reaching population "distributions" having strictly negative population in some eigenstates. This is indeed the part that makes things more involved.

Proof. In order to better highlight the key elements of the proof we treat only the case $\omega_{i j} \neq \omega_{a b}, \forall(i, j) \neq(a, b)$, the general case bearing no new concepts. Let us denote $\bar{A}=-i A$ and $\bar{B}=-i B$. Then (5) become:

$$
\left\{\begin{array}{l}
\frac{\partial}{\partial t} C_{\epsilon}=(\bar{A}+\epsilon(t) \bar{B}) C_{\epsilon} \\
C_{\epsilon}(t=0)=C_{0}
\end{array}\right.
$$


Denote by $c\left(\epsilon, C_{0}, t\right)=\left(c_{a}\left(\epsilon, C_{0}, t\right)\right)_{a=1}^{N}$ the solution at the time $t$ of (13) for the initial $(t=0)$ data $C_{0}$ and electric field $\epsilon(t)$.

We define the application $M: L^{2}(\mathbf{R}) \times \mathbf{R} \rightarrow \mathbf{R}^{N}$ given by

$$
M(\epsilon, t)=\left(\left|c_{a}\left(\epsilon, C_{0}, t\right)\right|^{2}\right)_{a=1}^{N}
$$

Note that by the necessary conditions (12) the range of $M$ is a subset of

$$
\left\{\left(x_{i}\right)_{i=1}^{N} \in \mathbf{R}^{N} ; \sum_{\left\{i ; \Psi_{i} \in V_{\alpha}\right\}} x_{i}=\sum_{\left\{i ; \Psi_{i} \in V_{\alpha}\right\}}\left|c_{0 i}\right|^{2}, \alpha=1, . ., K\right\}
$$

The system (13) can be written in the integral form:

$$
c(t)=e^{\bar{A} t} c(0)+\int_{0}^{t} e^{\bar{A}(t-s)} \epsilon(s) \bar{B} c(s) d s
$$

which gives us (cf. also [1]) the formula of the (Fréchet) derivative $D_{\epsilon} c\left(\epsilon, C_{0}, t\right)$ of $c\left(\epsilon, C_{0}, t\right)$ with respect to $\epsilon$ computed at $\epsilon(t) \equiv 0$ :

$$
\left.D_{\epsilon} c\left(\epsilon, C_{0}, t\right)\right|_{\epsilon=0} \cdot \tilde{\epsilon}=\int_{0}^{t} e^{\bar{A}(t-s)} \tilde{\epsilon}(s) \bar{B} e^{\bar{A} s} c(0) d s
$$

Denoting by $w(t)$ the free evolution of the system $\left(w_{a}(t)=c_{a}\left(0, C_{0}, t\right)\right.$ and $\left.w(t)=c\left(0, C_{0}, t\right)\right)$ and using the canonical base $\left\{e_{1}, \ldots, e_{N}\right\}$ of $\mathbf{R}^{N}$ we can write:

$$
\begin{array}{r}
\left.D_{\epsilon} M(\epsilon, t)\right|_{\epsilon=0} \cdot \tilde{\epsilon}=\left(D_{\epsilon} w_{a}(t) \cdot \tilde{\epsilon} \overline{w_{a}(t)}+w_{a}(t) \overline{D_{\epsilon} w_{a}(t) \cdot \tilde{\epsilon}}\right)_{a=1}^{N} \\
=\left[2 \operatorname{Re}\left(D_{\epsilon} w_{a}(t) \cdot \tilde{\epsilon} \overline{w_{a}(t)}\right)\right]_{a=1}^{N} \\
=\left[2 \operatorname{Re}\left(<D_{\epsilon} w(t) \cdot \tilde{\epsilon}, e_{a}>\overline{w_{a}(t)}\right)\right]_{a=1}^{N}
\end{array}
$$

Since

$$
\left(e^{\bar{A}(t-s)} \tilde{\epsilon}(s) \bar{B} e^{\bar{A} s}\right)_{a b}=e^{-i \lambda_{a}(t-s)} \tilde{\epsilon}(s)(-i) B_{a b} e^{-i \lambda_{b} s}
$$

and taking into account the explicit formula for $w_{a}(t)$

$$
w_{a}(t)=e^{-i \lambda_{a} t} w_{a}(0), a=1, \ldots, N
$$

one obtains first

$$
\left.D_{\epsilon} M(\epsilon, t)\right|_{\epsilon=0} \cdot \tilde{\epsilon}=\left[2 \operatorname{Re}\left(-i \sum_{b=1}^{N} \int_{0}^{t} e^{-i \lambda_{a} t} B_{a b} e^{i \omega_{a b} s} \tilde{\epsilon}(s) w_{a}(0) \overline{w_{b}(t)} d s\right)\right]_{a=1}^{N}
$$

and then

$$
\left.D_{\epsilon} M(\epsilon, t)\right|_{\epsilon=0} \cdot \tilde{\epsilon}=\left[2 \operatorname{Re}\left(-i \sum_{b=1}^{N} \int_{0}^{t} w_{b}(0) \overline{w_{a}(0)} B_{a b} e^{-i \omega_{a b}(t-s)} \tilde{\epsilon}(s) d s\right)\right]_{a=1}^{N}
$$


Armed with this formula we are ready to tackle with the local controllability problem. This is in fact a particular surjectivity property of $M(\epsilon, t)$. We will fix $t=T \neq 0$ and will prove that $D_{\epsilon} M$ has the surjectivity property we desire; by the implicit function theorem the conclusion will follow then for $M$ itself.

We prove that $D_{\epsilon} M(\epsilon, T)$ is onto the linear manifold (P) (product of hyper-planes of $\left.\mathbf{R}^{\text {cardinality }\left(S_{\alpha}\right)}, \quad \alpha=1, . ., K\right)$ :

$$
\left\{\left(x_{i}\right)_{i=1}^{N} \in \mathbf{R}^{N} ; \sum_{\left\{i ; \Psi_{i} \in V_{\alpha}\right\}} x_{i}=0, \alpha=1, . ., K\right\}
$$

whose $M(0, T)$-translation is tangent in $(0, T)$ to the range of $M$.

Denote by $f_{a}, a=1, \ldots, N$ the components of $D_{\epsilon} M$ :

$$
\left.D_{\epsilon} M(\epsilon, t)\right|_{\epsilon=0} \cdot \tilde{\epsilon}=\left(<f_{a}, \tilde{\epsilon}>_{L^{2}}\right)_{a=1}^{N}
$$

Due to the finite dimensionality of our setting we just have to show that the range of $\left.D_{\epsilon} M(\epsilon, t)\right|_{\epsilon=0}$ has a null orthogonal with respect to $(\mathrm{P})$, that is any vector $k=\left(k_{a}\right)_{a=1}^{N} \in \mathbf{R}^{N}$ such that

$$
\begin{array}{r}
\sum_{\left\{i ; \Psi_{i} \in V_{\alpha}\right\}} k_{i}=0, \quad \alpha=1, \ldots, K \\
\sum_{i=1}^{N} k_{i} \cdot<f_{i}, \tilde{\epsilon}>_{L^{2}}=0, \forall \tilde{\epsilon} \in L^{2}([0, T])
\end{array}
$$

is necessary the null vector.

The relation (22) can also be written $\sum_{i=1} k_{i} \cdot f_{i}(s)=0, \forall 0 \leq s \leq T$ or, in full format,

$$
\sum_{a, b=1}^{N} k_{a} B_{a b} \cdot 2 \operatorname{Re}\left[i w_{b}(0) \overline{w_{a}(0)} e^{-i \omega_{a b}(T-s)}\right] \equiv 0, \forall 0 \leq s \leq T
$$

Grouping together similar terms one gets for all $0 \leq s \leq T$

$$
\sum_{a<b}^{N}\left(k_{a}-k_{b}\right)\left|w_{a}(0)\right|\left|w_{b}(0)\right| B_{a b} \cdot 2 \operatorname{Re}\left[i \frac{w_{b}(0)}{\left|w_{b}(0)\right|} \frac{\overline{w_{a}(0)}}{\left|w_{a}(0)\right|} e^{-i \omega_{a b}(T-s)}\right]=0
$$

It suffices now to notice that since in $\left\{\omega_{a b} ; a<b\right\}$ there are no repetitions, the functions of $s$ are all incommensurable and of null sum. Therefore coefficients are all zero:

$$
\left(k_{a}-k_{b}\right)\left|w_{a}(0)\right|\left|w_{b}(0)\right| B_{a b}=0, a, b=1, \ldots, N, a<b
$$

Working on connected components of the transfer graph if follows that $k_{a}=$ const, for all $a$ such that $\Psi_{a} \in V_{\alpha}, \quad \alpha=1, . ., K$ which together with (21) implies that $k$ is the null vector, q.e.d. 
Remark 2 One can view this local controllability theorem as an encouraging argument when designing numerical algorithms. Indeed one is sure that a well designed algorithm will at least be able to improve the initial guess $\epsilon(t) \equiv 0$. It is however the next result that positively informs us about the possibility of solving such a control problem.

Remark 3 There is a interesting property of the control solutions that this result highlights: the possibility of synchronous control. The theorem states that when working with several independent quantum systems (each modeled by a connected component of the transfer graph $G$ ) one can control one of them without interfering with the others (or controlling at the same time the others also); this may give an indication about when control in liquid phase or in other cases of mixtures of systems (one of them being "principal") may be possible: when the systems have different spectral signatures (non-degenerate transitions); this may eventually allow us to choose the right "secondary" systems to accompany our target.

Remark 4 The fact that there will always be (at least locally) control solutions that may be chosen to solve (finitely many) other control problems at the same time with our main control problem is suggesting that there is a rich diversity (and hence multiplicity) among the control solutions; therefore for a particular solution $\epsilon(t)$ only a part of all the information contained in $\epsilon(t)$ is useful for reaching the target, all the rest being only some sort of noise. One illustration of how little information ( $N-1$ Fourier coefficients) may take to reach a population distribution is given in the proof of the next result.

\subsection{Global controllability}

Theorem 3 Under the hypothesis [H] the population distribution of the system (5) is controllable.

Proof. Let us use the variable substitution $w_{\epsilon k}(t)=e^{i \lambda_{k} t} c_{\epsilon k}(t), k=1, . ., N$. Then the equations (5) become:

$$
\left\{\begin{array}{l}
i \frac{\partial}{\partial t} w_{\epsilon k}(t)=\sum_{l \neq k} \epsilon(t) e^{i\left(\lambda_{k}-\lambda_{l}\right) t} B_{k l} w_{\epsilon l}(t), \quad k=1, . ., N \\
w_{\epsilon k}(t=0)=c_{0 k}, \quad k=1, . ., N
\end{array}\right.
$$

Since $\left|w_{\epsilon k}(t)\right|=\left|c_{\epsilon k}(t)\right|, \forall t \geq 0$ studying the controllability of (5) is equivalent to studying the controllability of (26). Regarding our definition of the controllability we understand that the goal is in fact to "rearrange" the population distributions inside each connected component of $G$ i.e. to transfer population among vertices belonging to the same connected component. 
Let us define an elementary population transfer of $\mu$ units between the eigenstate $\Psi_{k}$ and the eigenstate $\Psi_{l}$ by the final conditions

$$
\left\{\begin{array}{l}
\left|w_{\epsilon k}(T)\right|^{2}=\left|w_{\epsilon k}(0)\right|^{2}-\mu \\
\left|w_{\epsilon l}(T)\right|^{2}=\left|w_{\epsilon l}(0)\right|^{2}+\mu \\
\left|w_{\epsilon i}(T)\right|^{2}=\left|w_{\epsilon i}(0)\right|^{2}, \quad \forall i \neq k, l
\end{array}\right.
$$

Then it is easy to see that our problem can be decomposed into elementary population transfers between the eigenstates. In fact one can choose these transfers to happen between edges of the graph $G^{6}$ i.e. between states $\Psi_{k}$ and $\Psi_{l}$ such that $B_{k l} \neq 0$. Of course these population transfers need only be (arbitrary precise but) approximate.

Let us choose $\epsilon(t)$ of the form $\epsilon(t)=\frac{1}{p} r_{k l} \cos \left(\omega_{k l} t\right)=\frac{1}{p} r_{k l} \frac{e^{i \omega_{k l} t}+e^{-i \omega_{k l}} t}{2}$.

Then (26) can be written in the form:

$$
\begin{array}{r}
i \frac{\partial}{\partial t} w_{\epsilon a}(t)=\sum_{b \neq a} \epsilon(t) e^{i \omega_{a b} t} B_{a b} w_{\epsilon b}(t), \quad a=1, . ., N \\
i \frac{\partial}{\partial t} w_{\epsilon a}(t)=\sum_{b \neq a} \frac{1}{p} r_{k l} B_{a b} \frac{e^{i\left(\omega_{a b}+\omega_{k l}\right) t}+e^{i\left(\omega_{a b}-\omega_{k l}\right) t}}{2} w_{\epsilon b}(t) \quad a=1, . ., N
\end{array}
$$

One can see that the only terms that do contain non-oscillatory functions are $i \frac{\partial}{\partial t} w_{\epsilon k}(t)$ and $i \frac{\partial}{\partial t} w_{\epsilon l}(t)$ since in this case one gets in the second term quantities like $\frac{e^{i\left(\omega_{k l}-\omega_{k l}\right) t}}{2}=\frac{1}{2}$ or $\frac{e^{i\left(\omega_{l k}+\omega_{k l}\right) t}}{2}=\frac{1}{2}$. We will show that in the limit $p \rightarrow \infty$ for the final time $p T$ ( $\mathrm{T}=$ fixed) all other (oscillatory) terms can be neglected. Indeed let us replace $w_{\epsilon a}(t), a=1, \ldots, N$ by $U_{a}(t), a=1, \ldots, N$ given by:

$$
\left\{\begin{array}{l}
U_{k}(t)=\frac{w_{\epsilon k}(t)+w_{\epsilon l}(t)}{2} \cdot e^{i \frac{1}{2 p} r_{k l} B_{k l} t} \\
U_{l}(t)=\frac{w_{\epsilon k}(t)-w_{\epsilon l}(t)}{2} \cdot e^{i \frac{1}{2 p} r_{k l} B_{k l} t} \\
U_{a}(t)=w_{\epsilon a}(t), a=1, \ldots, N, a \neq k, l
\end{array}\right.
$$

Then the evolution system is now

$$
i \frac{\partial}{\partial t} U_{a}(t)=\sum_{b \neq a} \frac{1}{p} r_{k l} B_{a b} f_{a b}(t) U_{b}(t)
$$

where the functions $f_{a b}$ are sums of exponentials $e^{i q t}$ with $q$ having one of the forms $\omega_{a b}-\omega_{k l}, \omega_{a b}+\omega_{k l}, 2 \omega_{k l}, \omega_{a k}-\omega_{k l}+\frac{1}{2 p} r_{k l} B_{k l}, \ldots$ What is important

\footnotetext{
${ }^{6}$ Since each $G_{\alpha}$ is connected it contains at least a tree; one can prove recursively that in fact at most $N-1$ such operations are needed. Moreover since each $G_{\alpha}$ remains connected after having eliminated all degenerate transitions one can suppose the transitions correspond to edges which have not been eliminated.
} 
about the frequencies $q$ is that we are able to bound their absolute values by two constants $c_{1}, c_{2}>0$ that do not depend of $p$ as soon as $\mathrm{p}$ is large enough: $0<c_{1}<|q|<c_{2}<\infty$.

We will now show that for $p$ large enough $\left|U_{a}(p T)-U_{a}(0)\right|, a=1, \ldots, N$ is as small as we want ( $\mathrm{T}$ is fixed). Let us denote $g_{a b}=r_{k l} B_{a b} f_{a b}(t)$. Denote also by $G_{a b}(t)$ the primitive of $g_{a b}$ that is zero for $t=0$. From the form of the functions $f_{a b}$ we see that there exists a constant $C_{0}$ independent of $p$ such that $\left|G_{a b}(t)\right|<C_{0}, \forall t \in \mathbf{R}$. Then

$$
\begin{array}{r}
i U_{a}(p T)=i U_{a}(0)+\int_{0}^{p T} i \frac{\partial}{\partial t} U_{a}(t) d t=i U_{a}(0)+\int_{0}^{p T} \sum_{b \neq a} \frac{1}{p} g_{a b} U_{b}(t) d t \\
=i U_{a}(0)+\sum_{b \neq a} \frac{1}{p} G_{a b}(p T) U_{b}(p T)+i \int_{0}^{p T} \sum_{b \neq a} \frac{1}{p} G_{a b}(t) i \frac{\partial}{\partial t} U_{b}(t) d t \\
=i U_{a}(0)+\sum_{b \neq a} \frac{1}{p} G_{a b}(p T) U_{b}(p T)+\frac{i}{p} \int_{0}^{p T} \sum_{b, c=1}^{N} G_{a b}(t) \frac{1}{p} g_{b c} U_{c}(t) d t
\end{array}
$$

Using the fact that $G_{a b}, g_{a b}$ and $U_{a}(t)$ are bounded functions on $\mathbf{R}$ it follows that for each $\eta>0$ we can choose $p$ large enough such that $\mid i U_{a}(p T)-$ $i U_{a}(0) \mid<\eta$. After having replaced back the $U_{a}$ in the system we conclude that for $p$ large enough the solutions of the system (29) computed in $t=p T$ are as close as we want to the solutions of

$$
\left\{\begin{array}{l}
i \frac{\partial}{\partial t} w_{\epsilon k}(t)=\epsilon(t) e^{i \omega_{k l} t} B_{k l} w_{\epsilon l} \\
i \frac{\partial}{\partial t} w_{\epsilon l}(t)=\epsilon(t) e^{i \omega_{l k} t} B_{k l} w_{\epsilon k} \\
i \frac{\partial}{\partial t} w_{\epsilon a}(t)=0, a=1, \ldots, N, a \neq k, l
\end{array}\right.
$$

A straightforward analysis of the case $N=2$ proves now that one can realize any desired population transfer by tuning the coefficient $r_{k l}$ depending on how many "population units" are to be transfered between the eigenstates $\Psi_{k}$ and $\Psi_{l}$.

Remark 5 The hypothesis $(H)$ is verified in a large class of practical cases (see [10]). Moreover there are examples where the absence of this hypothesis makes the system not controllable. One can consider for instance the case of a system made up by two identical and independent sub-systems. It is obvious that using the same laser pulse one cannot obtain different results for the components. The hypothesis $(H)$ is here to prevent such correlations induced by the similarities in the spectral signatures to go unnoticed.

Remark 6 Even if our approach is constructive it is not entirely optimal; one can see that there are simple ways to reduce the time required to reach 
the target by constructing simultaneously elementary transfers. In order to formalize this one should optimize a distributed transport problem ${ }^{7}$ on the graph $G$. On the other side numerical results suggest us to conjecture that the $L^{2}$ norm of the field $\epsilon(t)$ realizing the transfer remains constant.

As an improvement of the result above one can prove the following

Theorem 4 Let $d_{0}$ be the population distribution associated to the initial state $C_{0}: d_{0}=\left(\left|c_{0 i}\right|\right)_{i=1, \ldots, N}$. Under the hypothesis [H] any population distribution $d=\left(d_{i}\right)_{i=1}^{N}$ such that $d_{i}>0, i=1, \ldots, N$ and that verifies the necessary conditions (12) can be exactly reached.

Proof. The proof combines the global approximate controllability with a slightly stronger form of the local controllability result. In order to simplify the presentation let us denote for any state $C$ by $d(C)$ its associated population distribution: $d(C)=\left(\left|C_{i}\right|\right)_{i=1}^{N}$. We will first assume the following

Lemma 2 Let $C_{l}$ be an initial state and $d_{l}=d\left(C_{l}\right)$. Suppose $d_{l i}>0, i=$ $1, \ldots, N$ and that the hypothesis $[H]$ is verified. Then there exists an open neighborhood $D_{l}$ of $d_{l}$ (on the surface of $\mathbf{R}^{N}$ given by the necessary conditions (12) endowed with the canonical topology) such that any population distribution $d$ in $D_{l}$ can be exactly reached from from any initial state $C_{i}$ with $d\left(C_{i}\right) \in D_{l}$.

In order to prove the theorem one applies the lemma above for $d$ and obtains an open neighborhood $D$. By the global result there exists a field that drives the system from the initial state $C_{0}$ to some state $C_{1}$ with $d\left(C_{1}\right) \in D$. Since $d$ is obviously in any of its open neighborhoods (and in particular in $D)$ it follows that there exists a field that drives $C_{1}$ to $d$. All that remains to be done is to "glue" those two parts together, obtaining thus an field that allows to exactly reach $d$ starting from $C_{0}$ (and passing by $d_{1}$ ).

Let us now prove the lemma (2). Note first that the local controllability result is uniform with respect to the initial state. Indeed, with the same notations as in the proof of theorem (2), note that $c(\epsilon, C, T)$ is of $C^{1}$ class with respect to $C$. Moreover we have proved that the (Fréchet) derivative of $(\epsilon, C, T) \rightarrow\left(\left|c_{a}(\epsilon, C, t)\right|^{2}\right)_{a=1}^{N}$ with respect to $\epsilon$ computed at $(0, C, T)$ is surjective. This allows us to apply the implicit function theorem and conclude that there exists a neighborhood $D_{C}$ of $C$ and a neighborhood (for the topology cited above) $D$ of $d$ such that from any initial state $C_{i} \in D_{C}$ one can exactly reach any population distribution in $D$.

\footnotetext{
${ }^{7}$ see $[3]$
} 
Suppose now that the lemma (2) is not true. Then there exists a sequence of states $C_{n}$ (with $d_{n}=d\left(C_{n}\right)$ ) and a sequence of distributions $d_{n}^{t}$ such that $d_{n} \rightarrow d, d_{n}^{t} \rightarrow d$ and $d_{n}^{t}$ cannot be exactly reached from $C_{n}$. Each $C_{n}$ is characterized by the set of phase factors (that can be safely supposed to be in $[0,2 \pi]^{N}$ ) and population distribution $d_{n}$. Since the set $[0,2 \pi]^{N}$ is compact one can extract from the sequence of phase factors one sub-sequence that is converging; toghether with $d_{n} \rightarrow d$ it follows that one can find a sub-sequence of $C_{n}$ that is converging to some state $C$ with $d(C)=d$. In conclusion there exists a sequence $C_{n_{k}} \rightarrow C(k \rightarrow \infty)$ of states and a sequence $d_{n_{k}}^{t} \rightarrow d$ of population distributions such that one cannot exactly reach $d_{n_{k}}^{t}$ from $C_{n_{k}}$ for any $k \geq 1$. This is obviously contradicting the uniform properties of the local result, q.e.d.

\section{Application to a five level system}

As an application of the results above we will study a five-level system presented in [14]. It will be seen that the controllability is easy to check by our method; we will take advantage of the constructive side of the theory to support the theoretical results in numerical simulations.

The matrix representation of the operators involved are:

$$
A=\left(\begin{array}{ccccc}
1.0 & 0 & 0 & 0 & 0 \\
0 & 1.2 & 0 & 0 & 0 \\
0 & 0 & 1.3 & 0 & 0 \\
0 & 0 & 0 & 2.0 & 0 \\
0 & 0 & 0 & 0 & 2.15
\end{array}\right), B=\left(\begin{array}{lllll}
0 & 0 & 0 & 1 & 1 \\
0 & 0 & 0 & 1 & 1 \\
0 & 0 & 0 & 1 & 1 \\
1 & 1 & 1 & 0 & 0 \\
1 & 1 & 1 & 0 & 0
\end{array}\right)
$$

One can deduce the following transfer graph $G$ :

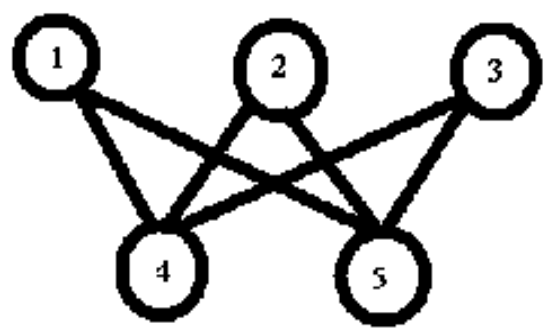

Since $G$ is obviously connected and since the hypothesis $(\mathrm{H})$ is also verified it follows that the population distribution of this system is controllable. We present some numerical examples of population transfer, drawing in each case 
the evolution of the populations $\left(\left|c_{i}(t)\right|^{2}, i=1, . ., 5\right)$ in the corresponding viewgraphs.

In the first case we want to transfer population from state 1 directly to state 5 . We write this control problem formally $(1,0,0,0,0) \rightarrow(0,0,0,0,1)$. Using a laser field of the form $\epsilon(t)=\beta \cos \left(\left(\lambda_{1}-\lambda_{5}\right) t\right)$ one obtains the following population evolution:

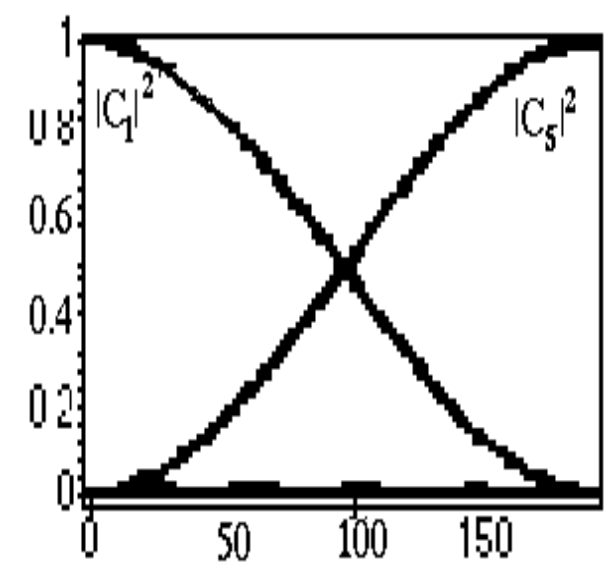

In the second example the target is the state 2 and we use state 4 as intermediary: $(1,0,0,0,0) \rightarrow(0,1,0,0,0)$. The laser takes now the form $\epsilon(t)=\beta_{1} \cos \left(\left(\lambda_{1}-\lambda_{4}\right) t\right)+\beta_{2} \cos \left(\left(\lambda_{4}-\lambda_{2}\right) t\right)$.

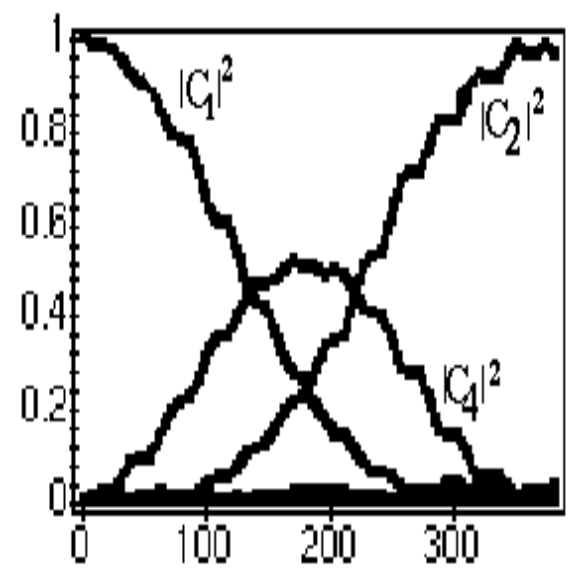

The last case is a cooperative control example where population is flowing to target state 4 from states 1 and $2:\left(\frac{1}{3}, \frac{2}{3}, 0,0,0\right) \rightarrow(0,0,0,1,0)$. The laser 
has the same general form.

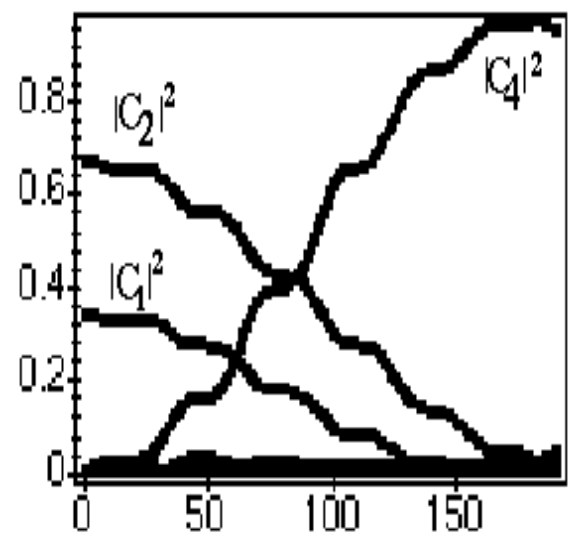

Remark 7 The transfer speed is essentially given by the matrix B. Studying the time needed for the three experiments one notes that for controls of the same order in $L^{\infty}$ norm the second is two times slower since the population has to go from $\Psi_{1}$ to $\Psi_{4}$ and then from $\Psi_{4}$ to $\Psi_{2}$; that could be also realized by two simulation of the first type.

Remark 8 With values in $O(1)$ in the $B$ matrix in order to obtain a precision of $10^{-2}$ our method require heuristically a " $p$ " of order $O\left(10^{2}\right)$ which is consistent (and of the same order of magnitude) with the time usually obtained in the literature (see [14]).

\section{Conclusions}

Controllability of the bilinear quantum systems has been studied in the infinite and finite dimensional settings. The infinite dimensional case has been seen to exhibit hidden restrictions due to some compacity properties of the equations involved. For the finite dimensional case and as long as one is interested in the populations of the eigenstates, positive results have been obtained for exact local and for global controllability. Easy to check and intuitively simple to understand necessary and sufficient conditions have been obtained to characterize the attainable set. Numerical simulations for a five level system are also presented.

Acknowledgements. It is a pleasure to acknowledge helpful talks that we had on this topic with Prof. Yvon Maday (ASCI Laboratory). 


\section{References}

[1] J.M.Ball, J.E.Madersen and M.Slemrod, "Controllability for distributed bilinear systems", SIAM J.Control and Optimization, vol 20, No.4, 1982, pp575-597

[2] P.Brumer and M.Shapiro, "Coherence Chemistry: Controlling Chemical Reactions with Lasers", Acc.Chem Res. Vol. 22, p.407 , 1989

[3] C.W.Churchmann, L.Ackoff, E.Arnoff, "Introduction à la recherche opérationnelle", Dunod, Paris, 1970

[4] M.Demilrap and H.Rabitz, Phys. Rew A., 47 2, p.831, 1983

[5] C.M. Dion et al., Chem. Phys.Lett 302(1999) 215-223

[6] C.M. Dion, A.Keller, O.Atabek \& A.D. Bandrauk, Phys. Rew. A 59(2) 1999, p.1382

[7] G.M.Huang, T.J.Tarn \& J.W.Clark, "On the controllability of quantummechanical systems", J.Math.Phys 24(1983), p.2608

[8] Mei Kobayashi," Mathematics make molecules dance" , SIAM News 24 (1998)

[9] A.P.Pierce, M.A. Dahleh and H.Rabitz, Phys Rev.A 37, 4950 (1998)

[10] V. Ramakrishna \& al. "Controllability of molecular systems" , Phys. Rew. A, Vol 51, No.2, 1995 p 960-966

[11] S.Shi and H.Rabitz, "Optimal control of selectivity of unimolecular reactions via an excited electronic state with designed lasers" , J.Chem Phys 97(1992), 276

[12] S.Shi, A.Woody, and H.Rabitz, "Optimal control of selective vibrational excitation in harmonic linear chain molecules" , J.Chem Phys. 88(1988), p. 6870

[13] D.J.Tannor and S.A.Rice, J.Chem Phys 83(1985), p.5013

[14] S.H. Tersigni, P.Gaspard and S.A. Rice, "On using shaped light pulses to control the selectivity of product formation in a chemical reaction: An application to a multiple level system" J.Chem Phys 93(1990),p.1670

[15] W.S.Warren, H.Rabitz and M.Dahleh, "Coherent control of quantum dynamics", Science 259 (1993) 


\section{Appendix}

Even if the physical interpretation for $\left|c_{i}(t)\right|^{2}, i=1, \ldots, N$ as populations is valid only in the case of a diagonal hamiltonian our results can be extended for general auto-adjoint internal hamiltonian matrix $H_{0}$ and dipole operator $B$.

Indeed since $H_{0}$ is auto-adjoint there exists a $(N \times N)$ unitary transform $U$ such that $U H_{0} U^{t}$ be diagonal. It is then straightforward to state the above results for the system so transformed.

What may be more interesting to notice is that $B_{i i}$ need not be zero in order for the controllability results to remain true. This is easy to prove for the local controllability result: the set of formulae (25) remains the same since for $a=b$ the term in the formula (23) becomes:

$$
k_{a} B_{a a} 2 \operatorname{Re}\left[i w_{a}(0) \overline{w_{a}(0)} e^{-i \omega_{a a} s}\right]=k_{a} B_{a a} 2 \operatorname{Re}\left[i\left|w_{a}(0)\right|^{2}\right]=0
$$

For the global controllability result one has to work with a modified variable substitution $w_{\epsilon k}(t)=e^{i\left(l_{k}+\epsilon(t) B_{k k}\right) t} c_{\epsilon k}(t), k=1, . ., N$. Since the $L^{\infty}$ norm of $\epsilon(t)$ is to be chosen small enough later in the proof, one realizes that in fact the uniform (in $p$ and $t$ ) boundeness of the $g_{a b}(t), G_{a b}(t)$ still holds true, which is enough to obtain the conclusion of the theorem. 